\title{
MODULAR REPRESENTATIONS OF METABELIAN GROUPS
}

BY

\section{B. G. BASMAJI}

ABSTRACT. The irreducible modular representations, the blocks, and the defect groups of finite metabelian groups are determined. Also the dimensions of the principal indecomposable modules are computed.

1. Introduction. Let $G$ be a finite metabelian group and $\Omega$ be an algebraically closed field with characteristic $p$ dividing the order $|G|$ of $G$. The purpose of this paper is to determine the irreducible representations over $\Omega$, the blocks ( $p$-blocks), the defect groups of $G$ and the dimensions of the indecomposable components of $\Omega G$. This is done by applying a number of fundamental results due to Brauer, found in Curtis-Reiner [6, Chapter XII], to the results of [1]. In $\$ 2$ we fix the notations. We prove a lemma in $\$ 3$ and apply it to determine the irreducible inequivalent modular representations of $G$ over $\Omega$. Assuming the knowledge of linear representations of some subgroups, we are able to give in $\$ 4$ the blocks and their defect groups. This makes it possible to determine all blocks containing a linear representation. We prove that all principal indecomposable modules belonging to a block have the same dimension and we compute this dimension. In $\$ 5$ we compute the decomposition and Cartan matrices of a block of the metacyclic group.

2. Notations. Let $G$ be a finite group and $A$ be a normal abelian subgroup of $G$ and assume $G / A$ is abelian. Let $W$ be a subgroup of $A$ and let $K(W) / W$ be a maximal abelian subgroup of $N(W) / W$ containing $A / W$ where $N(W)$ is the normalizer of $W$ in $G$. The subgroup $K(W)$ is not unique and can also be defined as a subgroup of $G$ containing $A$, the derived group $K(W)^{\prime} \subseteq W$; and $K(W)$ is maximal in the sense that if $K_{1}$ is a subgroup of $G$ containing $K(W)$ and $K_{1}^{\prime} \subseteq W$ then $K_{1}$ $=K(W)$. If $W_{1}$ is a subgroup of $W$, then for each $K\left(W_{1}\right)$ we can choose a $K(W)$ containing $K\left(W_{1}\right)$. If $W_{1}$ is conjugate to $W$ then we may let $K\left(W_{1}\right)=K(W)$. Also if $W_{0}=\bigcap_{x \in G^{x}} x^{-1} W x$ then we may choose $K\left(W_{0}\right)=K(W)$.

Let $H$ be a subgroup of $A$ such that $A / H$ is cyclic and let $z_{1} A, \cdots, z_{w} A$ be a basis of $K(H) / A$ with $z_{i} A$ of order $t_{i}$. Let $\bar{Q}$ be the algebraic closure of the field $Q$ of the rationals and let $T$ be a linear representation

Received by the editors August 27, 1971.

AMS 1970 subject classifications. Primary 20C15, $20 \mathrm{C} 20$.

Key words and phrases. Modular representations, blocks, defect groups, principal indecomposable modules, Brauer characters, decomposition matrices, Cartan matrices. 
(over $\bar{Q}$ ) of $A$ with kernel $H$. There exist $|K(H) / A|$ distinct representations $T^{\prime}$ which are extensions of $T$ to $K(H)$ and $T^{\prime}\left(z_{i}\right)^{t_{i}}=T\left(z_{i}^{t_{i}}\right)$. From [1] the induced representation $T^{\prime G}$ of $G$ is irreducible of degree $|G / K(H)|$. All the irreducible inequivalent representations of $G$ are given by the set of all $T^{\prime G}$ with $T^{\prime} \epsilon$ $\bigcup_{R_{c}}(H, K(H))$ where the union is over all nonconjugate $H$ such that $A / H$ is cyclic and $R_{c}(H, K(H))$ is a complete set of representatives of the conjugate classes of the set $R(H, K(H))$ of all extensions $T^{\prime}$ of every possible $T$ with kernel $H$. In this paper conjugacy will mean $G$-conjugacy unless stated otherwise.

If $S$ is a linear representation of a subgroup $K$ of $G, K \supseteq A$, then $S$ can be extended to a subgroup $K_{1} \supseteq K$ provided that $K_{1}^{\prime} \subseteq \operatorname{ker} S \cap A$. In this case there are $\left|K_{1} / K\right|$ distinct such extensions of $S$.

Let $p$ (fixed all through this paper) be a prime dividing the order $|G|$ of $G$ and let $\nu_{p}$ be a fixed valuation of $\bar{Q}$ extending the $p$-adic valuation of $Q, \nu_{p}(p)$ $=1$. Let $O$ be the local ring of $\nu_{p}$ in $\bar{Q}, p$ the corresponding unique maximal prime ideal, and $\Omega=O / p$. The residue class map of $O$ onto $\Omega$ is denoted by bar; $v \rightarrow \bar{v}$. We let $\overline{1}=1$ and $\overline{0}=0$. If $S$ is a representation of $G$ over $O$ and $S(g)=\left(v_{i j}\right), g \in G$, then the representation $\bar{S}$ of $G$ over $\Omega$ is defined by $\bar{S}(g)=$ $\left(\bar{v}_{i j}\right)$. If $S$ is a representation of a subgroup $K$ of $G$ over $O$ then $\bar{S} G=\overline{S^{G}}$, where in both cases the same coset representatives of $K$ in $G$ are taken. Note that the representations $T, T^{\prime}$, and $T^{\prime G}$ are over $O$ and thus $\bar{T}, \bar{T}^{\prime}$, and $\bar{T}^{\prime} G$ are defined. For convenience we let $\tau=\bar{T}, \tau^{\prime}=\bar{T}^{\prime}$, and $\tau^{\prime} G=\bar{T}^{\prime} G$, and the subscripts on $T$ are carried on to $\tau$ as in $\tau_{i}=\bar{T}_{i}$ and $\tau_{1 x}^{\prime}=\bar{T}_{1 x}^{\prime}$. Note that in all cases by $\bar{S}^{\prime}$ we mean $\overline{S^{\prime}}$. Representations over $\bar{Q}$ are called ordinary and will be denoted by capital Latin letters without bars, and those over $\Omega$ are called modular and will be denoted by capital Latin letters with bars or by small Greek letters.

The notations $S \sim Z$ and $\sigma \sim \zeta$ mean equivalence of these representations over $\bar{Q}$ and over $\Omega$ respectively. If $K$ is a subgroup of $G$ then $S_{K}$ and $\sigma_{K}$ mean the restrictions of $S$ and $\sigma$ respectively to $K$.

We make a note about Brauer characters that will be used several times in this paper. Let $S$ be a linear representation of a subgroup $K$ of $G, K \supseteq A$. Then there exists a linear representation $Z$ of $K$ such that $Z(k)=1$ if $k$ is a $p$-element and $Z(k)=S(k)$ if $k$ is $p$-regular. Let $\chi$ and $\psi$ be the characters of $S^{G}$ and $Z^{G}$ respectively. Then $\chi(g)=\psi(g)=0$ if $g \notin K$, and $\chi(k)=\psi(k)=$ $\Sigma_{x \in G / K} S\left(k^{x}\right)$ if $k$ is a $p$-regular element of $K$, where $x \in G / K$ means $x$ runs over a coset representative of $K$ in $G$ and $k^{x}=x^{-1} k x$. Thus the Brauer characters of $\bar{S}^{G}$ and $\bar{Z}^{G}$ are equal and by $[6,(82.7)], \bar{S}^{G}$ and $\bar{Z}^{G}$ have the same composition factors.

3. Irreducible representations. Assume the notations of $\$ 2$. We have 
Lemma. Let $p \nmid|A / H|$.

(a) $\tau^{\prime G}$ is an irreducible representation of $G$.

(b) Let $T_{1}^{\prime}$ be a linear representation of $K(H)$ with $\operatorname{ker} T_{1}^{\prime} \cap A=H_{1}$ conjugate to $H$. Then $\tau^{\prime} G \sim \tau_{1}^{\prime G}$ if and only if $\tau^{\prime}$ and $\tau_{1}^{\prime}$ are conjugate.

(c) Let $T_{1}^{\prime}$ be a linear representation of $K\left(H_{1}\right), \operatorname{ker} T_{1}^{\prime} \cap A=H_{1}, p \nmid\left|A / H_{1}\right|$, and $H_{1}$ not conjugate to $H$. Then $\tau^{\prime G}$ and $\tau_{1}^{\prime G}$ are inequivalent.

Proof. (a) Let $K=K(H)$. For $x \in G$ assume $\tau^{\prime}\left(k^{x}\right)=\tau^{\prime}(k)$ for all $k \in K$. Then $\tau^{\prime}\left(k^{-1} k^{x}\right)=1$ or $k^{-1} k^{x}=k^{-1} x^{-1} k x$ is in $\operatorname{ker} \tau^{\prime} \cap A=\operatorname{ker} T^{\prime} \cap A=H$. Thus $k^{-1} x^{-1} k x \in H$ and $x \in K$. Hence for each $x \notin K$ there exists at least one $k \in K$ such that $\tau^{\prime}\left(k^{x}\right) \neq \tau^{\prime}(k)$. Thus $K$ is the inertia group of $\tau^{\prime}$ and $\tau^{\prime}$ has $|G / K|$ distinct conjugates which we denote by $\tau_{x}^{\prime}, \tau_{x}^{\prime}(k)=\tau^{\prime}\left(k^{x}\right)$. Note that $\left(\tau^{\prime G}\right)_{K} \sim \sum_{x \in G / K} \tau_{x}^{\prime}$ where the summation is direct sum of representations. Assume $\tau^{\prime} G$ is reducible and let $\sigma$ be an (irreducible) composition factor of $\tau^{\prime G}$ such that $\tau^{\prime}$ is a composition factor of $\sigma_{K}$. By Clifford's theorem ([5, Theorem 1], $[6,(49.7)]), \sigma_{K}$ is equivalent to $e$ copies of $\sum_{x \in G / K} \tau_{x}^{\prime}$. Thus $\sigma \sim \tau^{\prime} G$ and $\tau^{\prime} G$ is irreducible. An elementary but considerably longer proof of (a) could also be given.

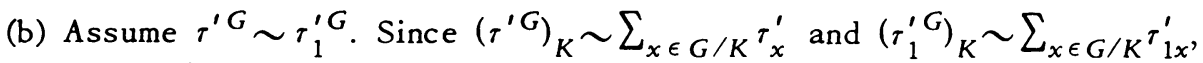
it follows that $r_{1}^{\prime}$ is conjugate $r^{\prime}$. The converse is easy.

(c) Let $K=K(H), K_{1}=K\left(H_{1}\right)$, and assume $|K|=\left|K_{1}\right|$, for otherwise there is nothing to prove. Let $m=|G / K|$ and let $G=x_{1} K \cup \cdots \cup x_{m} K=y_{1} K_{1} \cup \cdots$ $\cup y_{m} K_{1}$. For any $x_{j}$ and $y_{i}$ there exists $d \in A$ such that $d^{x_{j}} \in H$ and $d^{y_{i}} \notin H_{1}$. Assume $S=\left(v_{i j}\right)$ is an $m \times m$ matrix over $\Omega$ such that $S \tau^{\prime} G_{(g)=\tau_{1}^{\prime}}{ }_{(g) S}$ for all $g \in G$. Then, in particular, this is true for $g=d$ or $v_{i j} \tau^{\prime}\left(d^{x_{j}}\right)=\tau_{1}^{\prime}\left(d^{y_{i}}\right) v_{i j}$. Since $1=\tau^{\prime}\left(d^{x} j\right) \neq \tau_{1}^{\prime}\left(d^{y_{i}}\right)$ we have $v_{i j}=0$ and thus $S=0$ which proves the result.

Using the notations of $\S 2$ let $p \nmid|A / H|$ and $M(H, K(H))$ be the set of all $\tau^{\prime}$ where $T^{\prime} \in R(H, K(H))$. Let $M_{c}(H, K(H))$ be a complete set of representatives of the conjugate classes of $M(H, K(H))$.

Theorem 1. All the irreducible inequivalent representations of $G$ over $\Omega$ are given by the set of all $\tau^{\prime G}$ where $\tau^{\prime} \in \bigcup_{M_{c}}(H, K(H))$ and the union is over all nonconjugate $H, A / H$ cyclic, and $p \nmid|A / H|$.

Proof. The lemma implies that all the representations $\tau^{\prime G}$ in the theorem are irreducible and inequivalent. Thus the $p$-rank of the decomposition matrix is greater than or equal to $\left|\bigcup M_{c}(H, K(H))\right|$.

Any irreducible ordinary representation of $G$ is given by $T_{1}^{\prime G}$ where $T_{1}^{\prime}$ is a linear representation of $K\left(H_{1}\right)$ with $\operatorname{ker} T_{1}^{\prime} \cap A=H_{1}$. Now $H_{1}$ determines a unique subgroup $H$ of $A$ such that $H_{1} \subseteq H, p \nmid|A / H|$, and $H / H_{1}$ is a (cyclic) p-group. Pick $K(H) \supseteq K\left(H_{1}\right)$ and let $S$ be a linear representation of $K\left(H_{1}\right)$ such 
that $S(k)=1$ if $k$ is a $p$-element and $S(k)=T_{1}^{\prime}(k)$ if $k$ is $p$-regular. Now $\operatorname{ker} S \cap A=H$ and thus there exist $\left|K(H) / K\left(H_{1}\right)\right|$ extensions $S^{\prime}$ of $S$ to $K(H)$. Since $S_{K\left(H_{1}\right)}^{\prime}=S$, it follows that $S^{K(H)} \sim \sum S^{\prime}$ and thus $S^{G} \sim \sum S^{\prime}{ }^{\prime}$ where the summation (direct sum of representations) is over the $\left|K(H) / K\left(H_{1}\right)\right|$ extensions $S^{\prime}$ of $S$ to $K(H)$. Each $\bar{S}^{\prime}$ is conjugate to some $r^{\prime} \in M_{c}(H, K(H))$ and thus each $\bar{S}^{\prime} G$ is equivalent to some $\tau^{\prime} G$. From $\$ 2$, the Brauer character of $\bar{T}_{1}^{\prime G}$ is a sum of Brauer characters of some $\tau^{\prime G}$ with $\tau^{\prime} \in M_{c}(H, K(H))$. Thus the decomposition matrix has $p$-rank equal to $\left|\bigcup M_{c}(H, K(H))\right|$, and by $[6,(83.5)]$ the result follows.

Remark. Let $A / H$ be cyclic ( $p$ may divide $|A / H|$ ) and let $K_{1} / H$ and $K_{2} / H$ be two maximal abelian subgroups of $N(H) / H, K_{i} / H \supseteq A / H, i=1,2$. Set $K=$ $K_{1} \cap K_{2}$ and let $S$ be a linear representation of $K$ with $\operatorname{ker} S \cap A=H$. Let $S_{1}$ be an extension of $S$ to $K_{1}$. Then, as done in the proof of Theorem $1, S^{G} \sim \sum S_{1}^{G}$ where the summation is over the distinct $\left|K_{1} / K\right|$ extensions $S_{1}$ of $S$ to $K_{1}$. Similarly $S^{G} \sim \sum S_{2}^{G}$ where $S_{2}$ runs over the $\left|K_{2} / K\right|$ extensions of $S$ to $K_{2}$. Since $S_{1}^{G}$ and $S_{2}^{G}$ are irreducible, it follows that each $S_{1}^{G}$ is equivalent to some $S_{2}^{G}$ and conversely each $S_{2}^{G}$ is equivalent to some $S_{1}^{G}$. In particular $\left|K_{1}\right|=\left|K_{2}\right|$. Let $\chi$ be the character of $S_{1}^{G}$. Then $\chi$ vanishes outside $K$ and thus it depends only on $S$. Thus $S_{1}^{G} \sim S_{1}^{\prime G}$ for any two extensions $S_{1}$ and $S_{1}^{\prime}$ of $S$ to $K_{1}$. Therefore all $S_{1}^{G}$ and $S_{2}^{G}$ are equivalent. This implies that the Brauer characters of $\bar{S}_{1}^{G}$ and $\bar{S}_{2}^{G}$ are equal, and by $[6,(82.7)]$ they have the same composition factors. In particular if $p \nmid|A / H|$, then $\bar{S}_{1}^{G}$ and $\bar{S}_{2}^{G}$ are irreducible and thus equivalent.

4. Blocks. Let $p \nmid|A / H|, A / H$ cyclic, and let $\operatorname{Sub}(H)$ be the set of all subgroups $L$ of $H$ such that $A / L$ is cyclic and $H / L$ is a $p$-group. Let $L_{1}, \cdots, L_{t}$ be the nonconjugate minimal subgroups in $\operatorname{Sub}(H)$ and set $\Lambda=\bigcap_{i=1}^{t} L_{i}$ and $K=$ $K(\Lambda)$. If $\Lambda_{1}=\bigcap^{\prime} L$ and $\Lambda_{2}=\bigcap^{\prime}\left(\bigcap_{x \in G^{x}} x^{-1} L x\right)$ where the intersection $\bigcap^{\prime}$ is of all elements $L$ of $\operatorname{Sub}(H)$, then we may pick $K\left(\Lambda_{1}\right)=K\left(\Lambda_{2}\right)=K(\Lambda)=K$. For each $L \in \operatorname{Sub}(H)$ choose $K(L) \supseteq K$. Let $C(H, K(\Lambda)$ ) be a complete set of representatives of the conjugate classes of the set of all linear representations $\sigma$ of $K$ with $\operatorname{ker} \sigma \cap A=H$. Let $B(\sigma, H)$ be the set of all representations $T^{\prime G}$ where $T^{\prime}$ is a linear representation of $K(L), L \in \operatorname{Sub}(H)$, with $\operatorname{ker} T^{\prime} \cap A=L$ and $\bar{T}_{K}^{\prime}$ conjugate to $\sigma$. Include in $B(\sigma, H)$ the irreducible composition factors of $\bar{T}^{\prime} G$, $T^{\prime G} \in B(\sigma, H)$ and identify equivalent representations. Note that two irreducible representations $S$ and $Z$ are in the same block if and only if they are linked. We say $S$ and $Z$ are linked if there exist irreducible representations $S=S_{0}, S_{1}, \cdots$, $S_{n}=Z$ such that $\bar{S}_{i}$ and $\bar{S}_{i+1}$ have a composition factor in common for $i=0,1$, $\cdots, n-1$. We prove

Theorem 2. All the distinct blocks of $G$ are given by the collection of the sets $B(\sigma, H)$ where $H$ runs over all nonconjugate subgroups of $A, A / H$ cyclic, $p \nmid|A / H|$, and $\sigma$ runs over the elements of $C(H, K(\Lambda))$. 
Proof. If $L$ is any subgroup of $A$ such that $A / L$ is cyclic, then $L$ is conjugate to some element in $\operatorname{Sub}(H)$ for some $H$. From $\$ 2$ we may take $L \in \operatorname{Sub}(H)$. Thus any irreducible ordinary representation of $G$ is given by $S^{G}$ where $S$ is a linear representation of $K(L), \operatorname{ker} S \cap A=L$, with $L \in \operatorname{Sub}(H)$ for some $H$. From the proof of Theorem 1 we have the composition factors of $\bar{S}^{G}$ equivalent to representations $\tau^{\prime} G$ where $\tau^{\prime} \in M_{c}(H, K(H))$. Hence the set of all irreducible representations $S^{G}, S$ any linear representation of $K(L)$ with $\operatorname{ker} S \cap A=L$ and $L$ any element of Sub $(H)$, forms a collection of blocks. This means we only need to study this set of irreducible representations.

Let $1 \leq g \leq t, \Lambda_{g}=\bigcap_{i=1}^{g} L_{i}$ and let $\mu_{g}$ be a linear representation of $K\left(\Lambda_{g}\right)$ $\supseteq K$ such that $\mu_{g K}=\sigma$. Let $\operatorname{Rep}\left(\mu_{g}\right)$ be the set of all irreducible representations $S^{G}$ where $S$ is any linear representation of $K(L) \supseteq K\left(\Lambda_{g}\right)$, ker $S \cap A=L$, the restriction of $\bar{S}$ to $K\left(\Lambda_{g}\right)$ is conjugate to $\mu_{g}$, and $L$ is any element in $\operatorname{Sub}(H)$ with $L \supseteq L_{i}$ for some $i, 1 \leq i \leq g$. We use induction on $g$ to prove that any two elements of $\operatorname{Rep}\left(\mu_{g}\right)$ are linked, $1 \leq g \leq t$. Note that $\mu_{t}=\sigma$ and $\operatorname{Rep}(\sigma)$ forms the set of ordinary representations of $B(\sigma, H)$.

Assume $g=1$. Let $\mu=\mu_{1}$ and $J=K\left(L_{1}\right)$ and pick $K(L) \supseteq J$ whenever $L \epsilon$ $\operatorname{Sub}(H)$ and $L \supseteq L_{1}$. Let $\theta_{1}, \cdots, \theta_{m}$ be all the nonconjugate linear representations of $K(H) \supseteq J$ such that $\theta_{i J}=\mu, 1 \leq i \leq m$. Then $\theta_{i}^{G}, 1 \leq i \leq m$, are all irreducible and inequivalent. Let $Z$ be a linear representation of $J$, with $\operatorname{ker} Z \cap A$ $=L_{1}$ and $\bar{Z}$ conjugate to $\mu$. Then $Z^{G}$ is irreducible. Let $M$ be a linear representation of $J, M(k)=1$ if $k$ is a $p$-element and $M(k)=Z(k)$ if $k$ is $p$-regular. Since $\operatorname{ker} M \cap A=H$ we have $\left|K(H) / K\left(L_{1}\right)\right|$ extensions $M^{\prime}$ of $M$ to $K(H)$. Every $\bar{M}^{\prime}$ is conjugate to some $\theta_{i}$ and conversely every $\theta_{i}, 1 \leq i \leq m$, is conjugate to some $\bar{M}^{\prime}$. Also $M^{G} \sim \sum M^{\prime} G$ where the summation is over all the extensions $M^{\prime}$ of $M$ to $K(H)$. From the lemma every $\bar{M}^{\prime} G$ is equivalent to some $\theta_{i}^{G}$ and conversely every $\theta_{i}^{G}, 1 \leq i \leq m$, is equivalent to some $\bar{M}^{\prime} G$. Thus every $\theta_{i}^{G}, 1 \leq i$ $\leq m$, and no others, appears as a composition factor of $\bar{Z}^{G}$.

Now let $S$ be any linear representation of $K(L) \supseteq J, \operatorname{ker} S \cap A=L, \bar{S}_{J}$ conjugate to $\mu, L \supseteq L_{1}$, and $L \in \operatorname{Sub}(H)$. Take $K(H) \supseteq K(L)$. Define a linear representation $N$ of $K(L)$ such that $N(k)=1$ if $k$ is a p-element and $N(k)=S(k)$ if $k$ is $p$-regular. Using the same method as above, we have some $\theta_{i}^{G}$, and no others, appear as composition factors of $\bar{S}^{G}$. Thus $\bar{S}^{G}$ and $\bar{Z}^{G}$ have a composition factor in common. Since $S$ was arbitrary it follows that any two elements of $\operatorname{Rep}(\mu)$ are linked.

If $t=1$ then there is nothing more to prove.

Let $t>1,1<g \leq t, \Delta=\Lambda_{g}=\bigcap_{i=1}^{g} L_{i}$, and $\Gamma=\Lambda_{g-1}=\bigcap_{i=1}^{g-1} L_{i}$. Let $J=$ $K(\Delta) \supseteq K, E=K(\Gamma) \supseteq J$ and $F=K\left(L_{g}\right) \supseteq J$. Let $\mu$ be a linear representation of $J$ such that $\mu_{K}=\sigma$, i.e. $\mu=\mu_{g}$, and let $\eta$ and $\zeta$ be any linear representations of 
$E$ and $F$ respectively such that $\eta_{J}=\zeta_{J}=\mu$. (Note $\eta=\mu_{g-1}$.) Define the set $\operatorname{Rep}(\zeta)$ as done above for $\mu_{1}$, i.e. by replacing $\zeta$ for $\mu_{1}$ and $L_{g}$ for $L_{1}$. Let the sets $\operatorname{Rep}(\mu)$ and $\operatorname{Rep}(\eta), \mu=\mu_{g}$ and $\eta=\mu_{g-1}$, be as in the beginning of this proof. From the above any two elements of $\operatorname{Rep}(\zeta)$ are linked. Assume any two elements of $\operatorname{Rep}(\eta)$ are linked. We shall construct two equivalent irreducible representations of $G$ one belonging to $\operatorname{Rep}(\eta)$ and the other to $\operatorname{Rep}(\zeta)$. Since $\eta$ and $\zeta$ were arbitrarily chosen provided that $\eta_{J}=\zeta_{J}=\mu$, this will prove that any two elements of $\operatorname{Rep}(\mu)$ are linked, which completes the induction process defined above.

Using the notations of the preceding paragraph, let $K_{1} / H$ and $K_{2} / H$ be maximal abelian subgroups of $N(H) / H$ with $K_{1} \supseteq E$ and $K_{2} \supseteq F$. Moreover, let $R=$ $K_{1} \cap K_{2}, C=R \cap E, D=R \cap F, V=D C$, and $W=R E$. Let $M$ be a linear representation of $J$ with $\operatorname{ker} M \cap A=H$ and $\bar{M}=\mu$. From the definitions of $E$ and $F$ we have $E \cap F=C \cap D \doteq J$. Thus there exist bases $x_{1} J, \cdots, x_{n} J$ of $C / J$ and $y_{1} J, \cdots, y_{m} J$ of $D / J$ such that $V / J=\left\langle x_{i} J, y_{j} J \mid 1 \leq i \leq n, 1 \leq j \leq m\right\rangle$ and $\left\langle x_{i} J\right\rangle \cap\left\langle y_{j} J\right\rangle=J / J$ for $1 \leq i \leq n, 1 \leq j \leq m$. This implies that there exists an extension $M^{\prime}$ of $M$ to $V$ such that $\bar{M}_{C}^{\prime}=\eta_{C}$ and $\bar{M}_{D}^{\prime}=\zeta_{D}$. Let $N$ be an extension of $M^{\prime}$ to $R$. Since $W / R \cong E / C$, there exists a basis $z_{1} R, \cdots, z_{s} R$ of $W / R$ such that $z_{1} C, \cdots, z_{s} C$ is a basis of $E / C$ and the orders of $z_{i} R$ in $W / R$ and $z_{i} C$ in $E / C$ are equal. Thus there exists an extension $Z$ of $N$ to $W$ such that $\bar{Z}_{E}=\eta$. Let $Z^{\prime}$ be any extension of $Z$ to $K_{1}$. Then $Z^{\prime} G$ and $\bar{Z}^{\prime} G$ are irreducible and $Z^{\prime} G \in \operatorname{Rep}(\eta)$. Similarly we can find an extension $Y^{\prime}$ of $N$ to $K_{2}$ such that $\bar{Y}_{F}^{\prime}$ $=\zeta$. Again $Y^{\prime} G$ and $\bar{Y}^{\prime} G$ are irreducible and $Y^{\prime} G \in \operatorname{Rep}(\zeta)$. From the remark in $\$ 3$ it follows that $Y^{\prime G}$ and $Z^{\prime G}$ are equivalent, and thus any two elements of $\operatorname{Rep}(\mu)$ are linked. Now the inductive hypothesis implies that any two ordinary representations in $B(\sigma, H)$ are linked.

Let $\mu_{1}, \cdots, \mu_{m}$ be the set of all nonconjugate linear representations of $K(H) \supseteq K(\Lambda)=K$ such that $\mu_{i K}=\sigma$. Let $T^{\prime G} \in B(\sigma, H), T^{\prime}$ as in first part of this section. Let $S$ be a linear representation of $K(L)$ such that $S(k)=1$ if $k$ is a $p$-element and $S(k)=T^{\prime}(k)$ if $k$ is $p$-regular. Then $\operatorname{ker} S \cap A=H$ and $S^{G} \sim$ $\sum S^{\prime} G$ where the summation is over all extensions $S^{\prime}$ of $S$ to $K(H) \supseteq K(L) \supseteq K$. Since $\bar{S}_{K}^{\prime}$ is conjugate to $\sigma$ it follows that only representations $\mu_{i}^{G}$ appear as composition factors of $\bar{T}^{\prime} G$. Since for each $\mu_{i}$ there exists a linear representation $S$ of $K(H)$ such that $\bar{S}=\mu_{i}$, it follows that $\mu_{i}^{G}, i=1, \cdots, m$, are the only modular representations in $B(\sigma, H)$. Now consider some $B\left(\sigma_{1}, H_{1}\right)$ and let $\zeta_{j}^{G}$, $j=1, \cdots, n$, be its modular representations where $\zeta_{1}, \cdots, \zeta_{n}$ are all the nonconjugate linear representations of $K\left(H_{1}\right) \supseteq K\left(\Lambda_{1}\right)=K_{1}$ such that $\zeta_{j K_{1}}=\sigma_{1}$. Here $\Lambda_{1}$ is defined for $H_{1}$ as $\Lambda$ was done for $H$. Now if $H_{1}$ is not conjugate to $H$ or $\sigma_{1}$ is not conjugate to $\sigma$ then $\mu_{i}^{G}$ and $\zeta_{j}^{G}$ are inequivalent. If $Z$ is an 
irreducible representation of $G$ and $Z \notin B(\sigma, H)$, then $Z \in B\left(\sigma_{1}, H_{1}\right)$ with either $H_{1}$ not conjugate to $H$ or $\sigma_{1}$ not conjugate to $\sigma$. Thus only representations $\zeta_{j}^{G}$ appear as composition factors of $Z$ or $Z$ is not linked to any element of $B(\sigma, H)$. Hence $B(\sigma, H)$ is a block which completes the proof of the theorem.

Note that the blocks $B(\sigma, H)$ where $H \supseteq G^{\prime}, G^{\prime}$ the derived group of $G$, are the only blocks of $G$ containing linear representations. If we set $A=G^{\prime}$ then these blocks are simply given by $B\left(\sigma, G^{\prime}\right)$. By Brauer [4, Proposition (4E)] these blocks are flat (as defined in [4]).

Now consider $K(H) \supseteq K(\Lambda)=K$. Since $K(H)$ is metabelian and $A$ is a normal subgroup of $K(H)$, the blocks of $K(H)$ are given by $b=b(\mu, \bar{H})$ where $\bar{H}$ runs over all nonconjugate (in $K(H)$ ) subgroups of $A$ such that $A / \bar{H}$ is cyclic and $p \nmid|A / \bar{H}|$. Let $\omega_{b}$ be the corresponding linear character of the center $Z(\Omega K(H))$ of $\Omega K(H)$. Consider the block $B=B(\sigma, H)$ of $G$ and let $\omega_{B}$ be the corresponding linear character of $Z(\Omega G)$.

Assume $\bar{H}$ is conjugate (in $G$ ) to $H$ and define $\bar{\Lambda}$ for $\bar{H}$ as $\Lambda$ was done for $H$. Take $K(\bar{\Lambda})=K(\Lambda)=K$ and assume $\mu$ is conjugate to $\sigma$. There exists a linear representation $S$ of $K(H)$ in $b=b(\mu, \bar{H})$ such that $S(k)=1$ if $k$ is a $p$ element, $\bar{S}_{K}=\mu$, and $\operatorname{ker} S \cap A=\bar{H}$. Now $S^{G}$ is irreducible and $S^{G} \in B=B(\sigma, H)$. Using the characters $\chi$ and $\chi^{G}$ of $S$ and $S^{G}$ respectively it is easy to show that $\omega_{b}^{G}=\omega_{B}$ and thus $b^{G}$ is defined and $b^{G}=B$. (The definitions of $\omega_{b}^{G}$ and $b^{G}$ can be found in $[2, \$ 2]$ or $[3, \$ 2,7]$ and need not be confused with induced representations.) Also, by Brauer [3, (4D)], $B$ covers $b$. Assume a block $b_{1}$ of $K(H)$ is covered by $B$, then by $[3,(4 A)]$ some constituent of $\left(S^{G}\right)_{K(H)}$ belongs to $b_{1}$. Thus we have

Corollary. Let $K(H) \supseteq K(\Lambda)$. The blocks $b=b(\mu, \bar{H})$ of $K(H)$ with $\mu$ conjugate to $\sigma$ and $\bar{H}$ conjugate to $H$ are the only blocks of $K(H)$ such that $b^{G}=$ $B(\sigma, H)$. Furthermore, these are the only blocks of $K(H)$ covered by $B(\sigma, H)$.

Now since $b(\sigma, H)$ contains a linear representation $S$ of $K(H)$ with $\bar{S}_{K}=\sigma$ and $\operatorname{ker} S \cap A=H$, it follows that any $p$-Sylow subgroup of $K(H)$ can be taken as a defect group of $b(\sigma, H)$. Since $S^{G}$ is an irreducible representation and $S^{G} \epsilon$ $B(\sigma, H)$ it follows that the defect $d$ of $B(\sigma, H)$ is given by $p^{d} \||K(H)|$. Thus from Brauer [2, (2B)] we have

Corollary. Let $K(H) \supseteq K(\Lambda)$. The defect group of $B(\sigma, H)$ is the p-Sylow subgroup of $K(H)$.

A principal indecomposable module belongs to a block $B$ if all its composition factors afford (modular) representations in $B$.

Theorem 3. The dimension of any principal indecomposable module belonging to the block $B(\sigma, H)$ is $p^{a}|G / K(H)|$ where $p^{a} \||K(H)|$. 
Proof. Let $L \in \operatorname{Sub}(H)$ and let $\mu^{G} \in B(\sigma, H)$ where $\mu$ is a linear representation of $K(H) \supseteq K(L) \supseteq K(\Lambda)=K$ with $\mu_{K}=\sigma$. Set $K(L)=J$ and $p^{l}=|H / L|$.

Let $T$ be a linear representation of $A$ with $\operatorname{ker} T=L$ and $\bar{T}=\mu_{A}$. Then there exist $\phi\left(p^{l}\right)$ such representations $T$ where $\phi$ is Euler's function.

Let $T^{\prime}$ be an extension of $T$ to $J$ such that $\bar{T}^{\prime}=\mu_{J}$ and let $p^{j} \||J / A|$. For each $T$ there are $p^{j}$ such extensions $T^{\prime}$.

Let $\nu(L)$ be the number of conjugates of $L$ in $\operatorname{Sub}(H)$. Then there are $\nu(L) p^{j} \phi\left(p^{l}\right)$ representations $T^{\prime}$ of $J$ such that $\operatorname{ker} T^{\prime} \cap A=L_{1} \in \operatorname{Sub}(H), L_{1}$ is conjugate to $L$, and $\bar{T}^{\prime}=\mu_{J}$.

Let $I=I\left\{\mu_{J}\right\}$ be the inertia group of $\mu_{J}$ in $G$. Then $I \supseteq K(H)$. Thus, since the inertia group of $T^{\prime}$ is $J$, there are

$$
g_{L}=\nu(L) p^{j} \phi\left(p^{l}\right) /|I / J|
$$

conjugate classes in the set of all representations $T^{\prime}$ defined above.

Now fix a representation $T^{\prime}$ satisfying the above conditions. Let $M$ be a representation of $J$ such that $M(k)=1$ if $k$ is a $p$-element and $M(k)=T^{\prime}(k)$ if $k$ is $p$-regular. Then $\bar{M}^{G}$ and $\bar{T}^{\prime G}$ have the same composition factors. Also $M^{G} \sim$ $\sum M^{\prime G}$ where the summation is over all the $|K(H) / J|$ extensions $M^{\prime}$ of $M$ to $K(H)$. Let $p^{s} \||K(H) / J|$; then there are $p^{s}$ extensions $M^{\prime}$ such that $\bar{M}^{\prime}=\mu$. Note that for all $M^{\prime}, \bar{M}_{J}^{\prime}=\mu_{J}=\bar{M}$. Since $K(H)$ is the inertia group of $\mu$ it follows that the number of linear representations $\theta$ of $K(H)$ such that $\theta$ is conjugate to $\mu$ and $\theta_{J}=\mu_{J}$ is $|I / K(H)|$. This implies that the multiplicity in which $\mu^{G}$ appears in the composition factors of $\bar{T}^{\prime G}$ is $m_{L}=p^{s}|I / K(H)|$.

Combining the above results it follows that the part of the column of the decomposition matrix $D$ corresponding to $\mu^{G}$ and the above representations $T^{\prime} G$ is $m_{L} I\left(g_{L}\right)$ where $I\left(g_{L}\right)$ is the $\left(g_{L} \times 1\right)$ matrix ${ }^{t}(1, \cdots, 1)$. (The part of this column corresponding to the remaining representations that we get from $K(L)$ and $L$ is zero.)

Let $U$ be a principal indecomposable module such that $U / U^{\prime}$ affords $\mu^{G}$ where $U^{\prime}$ is the unique maximal submodule of $U$. From $[6,(84.4)]$ the dimension of $U$ is

$$
u=\sum^{\prime} m_{L} g_{L}|G / J|
$$

where the summation $\sum^{\prime}$ is over all nonconjugate elements $L$ of Sub $(H)$. A short computation gives

$$
u=p^{a-a}\left|G_{i} K(H)\right| \sum \phi\left(p^{l}\right)
$$

where $p^{a} \||A|$ and the summation is over all elements of Sub $(H)$. The proof will be complete if we show $\sum \phi\left(p^{l}\right)=p^{a}$. 
Since $p \nmid|A / H|$ we have $p^{a} \||H|$. Let $H=Q \times P$ with $|P|=p^{a}$ and $p \nmid|Q|$. Then $H / L$ is a $p$-group if and only if $L \supseteq Q$. If $H / L$ is a $p$-group then $H / L$ is cyclic if and only if $A / L$ is cyclic. Thus without loss of generality we may take $H=P$ and $Q=1$. Now $\sum \phi\left(p^{4}\right)=p^{a}$ follows from the formula in $[1, \S 3]$ which completes the proof.

Corollary. Let $u$ be the dimension of a principal indecomposable module. Then $\nu_{p}(u)=\nu_{p}(|G|)$.

Since every modular representation in $B(\sigma, H)$ has the same degree $|G / K(H)|$ we have

Corollary. The dimension of the unique two-sided indecomposable ideal of $\Omega G$ that corresponds to $B(\sigma, H)$ is $b p^{a}|G / K(H)|^{2}$ where $p^{a} \||K(H)|$ and $b$ is the number of the (inequivalent) modular representations in $B(\sigma, H)$.

By block some authors mean the two-sided ideal in the above corollary. (See [61.)

5. Metacyclic groups. Consider the group

$$
G=\left\langle a, b \mid a^{n}=b^{m}=1, a^{k}=b^{t}, b^{-1} a b=a^{r}\right\rangle
$$

with $r^{t}-1 \equiv k r-k \equiv 0(\bmod n)$ and $t \mid m$. Set $A=\langle a\rangle$. For $x \mid n$ let $t_{x}$ be the smallest positive integer such that $r^{t_{x}} \equiv 1(\bmod x)$. Let $H_{x}=\left\langle a^{x}\right\rangle$ and $K_{x}=$ $K\left(H_{x}\right)=\left\langle a, b^{t_{x}}\right\rangle$. Let $n=p^{a} n^{\prime}, t=p^{\delta} t^{\prime}$ and $t_{x}=p^{\delta} t_{x}^{\prime}$, where $\left(p, n^{\prime}\right)=\left(p, t^{\prime}\right)=$ $\left(p, t_{x}^{\prime}\right)=1$. We have $\left|G / K_{x}\right|=t_{x}=p^{\delta x} t_{x}^{\prime}$ and $\left|K_{x} / A\right|=t / t_{x}=p^{\delta-\delta_{x}} t^{\prime} / t_{x}^{\prime}$. If $z \mid x$ then $t_{z}\left|t_{x}, t_{z}^{\prime}\right| t_{x}^{\prime}$, and $\delta_{z} \leq \delta_{x}$.

Let $s \mid n^{\prime}$ and let $\tau_{s}$ be a linear representation of $A$ such that $\tau_{s}(a)=\zeta_{s}^{y}$, where $\zeta_{s}$ is a primitive sth root of unity in $\Omega$ and $(y, s)=1$. Note that $\operatorname{ker} \tau_{s}=$ $H_{s}$. For each $\tau_{s}$ there exist $t^{\prime} / t_{s}^{\prime}$ representations $\tau_{s}^{\prime}$ of $K_{s}$ such that $\tau_{s A}^{\prime}=\tau_{s}$. Here $\tau_{s}^{\prime}\left(b^{t} s\right)^{t^{\prime} / t_{s}^{\prime}}=\tau_{s}\left(a^{k}\right)$. Each $\tau_{s}^{\prime G}$ is irreducible. To get all the irreducible inequivalent representations of $G$ over $\Omega$ we let $s$ run over all positive divisors of $n^{\prime}, y \in M_{s} / R_{s}$, and $\tau_{s}^{\prime}$ over all the $t^{\prime} / t_{s}^{\prime}$ representations with $\tau_{s A}^{\prime}=\tau_{s}$. Here $M_{s}$ is the multiplicative group of the reduced residues $(\bmod s)$ and $R_{s}$ is the subgroup of $M_{s}$ generated by $r$. For each $s$ we have $t^{\prime} \phi(s) / t_{s} t_{s}^{\prime}$ inequivalent modular representations $r_{s}^{\prime} G$ where $\phi$ is Euler's function.

Let $z=p^{\alpha} s$ and let $\tau_{z}=\tau_{s}$. Let $\sigma_{z}$ be a linear representation of $K=K_{z}$ such that $\sigma_{z A}=\tau_{z}$. Then $B\left(\sigma_{z}, H_{s}\right)$ is a block of $G$. We get different blocks by letting $s$ and $y$ run over the same values as above and $\sigma_{z}$ run over the $t^{\prime} / t_{z}^{\prime}$ distinct representations such that $\sigma_{z A}=\tau_{z}$. Thus for each $s$ we have $t^{\prime} \phi(s) / t_{s} t_{z}^{\prime}$ distinct blocks. Each block $B\left(\sigma_{z}, H_{s}\right)$ contains $b^{(s)}=t_{z}^{\prime} / t_{s}^{\prime}$ irreducible modular representations. These are given by $\mu_{i}^{G}, 1 \leq i \leq b^{(s)}$, where $\mu_{i}$ is a linear representation of $K_{s}$ with $\mu_{i K}=\sigma_{z^{*}}$ 
Fix $s$ and $\sigma_{z}$ and let $\sigma=\sigma_{z}, H=H_{s}$, and $b=b^{(s)}$. We shall give the decomposition and Cartan matrices of the block $B(\sigma, H)$. Let $\mu_{1}, \cdots, \mu_{b}$ be as above. Set $s_{i}=$ $p^{i} s, t_{s_{i}}=f_{i}, \delta_{s_{i}}=\beta_{i}, H_{s_{i}}=L_{i}$ and $F_{i}=K\left(L_{i}\right)=\left\langle a, b^{f_{i}}\right\rangle$ where $i=0,1, \cdots, \alpha$.

Note that $s_{0}=s, s_{a}=z, t_{s}=f_{0}, t_{z}=f_{a}, \delta_{s}=\beta_{0}$, and $\delta_{z}=\beta_{a}$. Also $K_{s}=F_{0}$ $\supseteq F_{1} \supseteq \cdots \supseteq F_{a}=K_{z}=K$ where $F_{i} / F_{j}$ is of order $f_{j} / f_{i}, \alpha \geq j \geq i \geq 0$.

Let $\lambda_{i}=t_{p}, \lambda_{1}=t_{p}$, then $r \equiv r_{1}{ }_{2}\left(\bmod p^{i}\right)$ where $r_{1}^{\lambda_{1}} \equiv 1\left(\bmod p^{i}\right)$ and $r_{2}^{p^{w}} \equiv 1\left(\bmod p^{i}\right), \lambda_{1} \mid p-1,0 \leq w<i$. Assuming $w$ is a smallest such positive integer we have $\lambda_{i}=\lambda_{1} p^{w}$. Thus $\lambda_{i+1} / \lambda_{i}=p$ or 1 for $i \geq 1$. But for $i \geq 1, f_{i}=$ $1 \mathrm{~cm}\left[\lambda_{i}, f_{0}\right]$ which proves that $f_{i+1} / f_{i}=p$ or 1 for $i \geq 1$. Since $f_{1} / f_{0}$ divides $\lambda_{1}$ it follows that $f_{1} / f_{0}$ divides $p-1$ and $\beta_{1}=\beta_{0}$. Note that $f_{i} / f_{1}$ divides $p^{i-1}, 1 \leq i \leq \alpha$. Since $t_{z} / t_{s}=f_{a} / f_{0}=\left(f_{a} / f_{1}\right)\left(f_{1} / f_{0}\right)$ we have the number of modular representations in $B(\sigma, H)$ equal to $b=t_{z}^{\prime} / t_{s}^{\prime}=f_{1} / f_{0}$ and thus $b \mid p-1$. Since $\left|F_{i} / A\right|=t / f_{i}$ it follows that the highest power of $p$ dividing $t / f_{i}$ is $p-\beta_{i}$ $=p^{\delta-\delta_{z}} f_{a} / f_{i}, \quad i \geq 1$.

Fix $F_{i}, i \geq 1$. There exist $\phi\left(p^{i}\right)$ linear representations $T$ of $A$ with $\operatorname{ker} T$ $=L_{i}$ and $\bar{T}=\sigma_{A}$. These partition into $f_{0} \phi\left(p^{i}\right) / f_{i}$ conjugate classes. Since $f_{a} / f_{i}$ is a power of $p$, it follows that each $T$ has $p^{\delta-\beta_{i}}=p^{\delta-\delta_{z}}\left(f_{a} / f_{i}\right)$ extensions $T^{\prime}$ to $F_{i}$ such that $\bar{T}_{K}^{\prime}=\sigma$. Thus there exist exactly

$$
b_{i}=p^{\delta-\delta} f_{a} f_{0} \phi\left(p^{i}\right) / f_{i}^{2}
$$

nonconjugate linear representations $T^{\prime}$ of $F_{i}$ such that $\bar{T}_{K}^{\prime}=\sigma$. The representations $T^{\prime G}$ are irreducible and inequivalent and belong to $B(\sigma, H)$. Using the Brauer character of $\bar{T}^{\prime G}$ and the method of this paper, it follows that every $\mu_{j}^{G}$, $j=1, \cdots, b$, appears with a multiplicity $f_{i} / f_{1}$ as composition factors of $\bar{T}^{\prime} G$.

For $i=0$ we have $b_{0}=p^{\delta-\delta_{s}}\left(f_{1} / f_{0}\right)=p^{\delta-\delta_{s}}$ nonconjugate representations $T^{\prime}$ of $K_{s}=F_{0}$ such that $\bar{T}_{K}^{\prime}=\sigma$. Each $\bar{T}^{\prime G}$ is irreducible. Thus for each $\mu_{j}^{G}$, $j=1, \cdots, b$, there are $b_{0} / b=p^{\delta-\delta_{s}}$ representations $T^{\prime G}$ such that $\bar{T}^{\prime G} \sim \mu_{j}^{G}$. The number of the ordinary representations in $B(\sigma, H)$ is $\sum_{i=0}^{a} b_{i}$.

Using the above and arranging the representations $T^{\prime}{ }^{i=0}$ and $\mu_{j}^{G}$ appropriately, the transpose of the decomposition matrix $D$ of $B(\sigma, H)$ is given by

$$
{ }^{t} D=\left({ }^{t} D_{0},{ }^{t} D_{1}, \ldots,{ }^{t} D_{a}\right)
$$

where

$$
D_{0}=\operatorname{diag}\left(I\left(b_{0} / b\right), \cdots, I\left(b_{0} / b\right)\right)
$$

and

$$
D_{i}=f_{i} / f_{1}\left(I\left(b_{i}\right), \ldots, I\left(b_{i}\right)\right), \quad i \geq 1
$$

with $I(x)$ the $(x \times 1)$ matrix ${ }^{t}(1, \cdots, 1)$ and $D_{i}, 0 \leq i \leq \alpha$, has $b$ columns. The Cartan matrix is given by 


$$
C={ }^{t} D \circ D=\sum_{i=0}^{a}{ }^{t} D_{i} \circ D_{i}=p^{\delta-\delta_{s}} E_{b}+\sum_{i=1}^{a}\left(f_{i} / f_{1}\right)^{2} b_{i}(l(b), \ldots, I(b))
$$

where $E_{b}$ is the $b \times b$ identity matrix. A short computation gives $\operatorname{det} C=$ $\left(p^{\delta-\delta} s\right)^{b} p^{a}$ which is a power of $p$ as expected from [6, (84.17)].

Let $U_{j}$ be a principal indecomposable module such that $U_{j} / U_{j}^{\prime}$ affords $\mu_{j}^{G}$ where $U_{j}^{\prime}$ is the maximal submodule of $U_{j}$. Then $U_{j}$ is of dimension $p^{\delta-\delta_{s}+a_{t}}{ }_{s}$. This could also be computed using the Cartan matrix above. In the composition factors of $U_{j}, p^{\delta-\delta} s\left[1+\left(p^{a}-1\right) / b\right]$ factors appear that afford $\mu_{j}^{G}$ and for each $q \neq j, 1 \leq q \leq b, p^{\delta-\delta} s\left(p^{a}-1\right) / b$ factors appear that afford $\mu_{q}^{G}$.

In particular if $p=2$ then $b=1$ and each block of $G$ contains one irreducible modular representation, and thus the two-sided ideal of $\Omega G$ that corresponds to $B(\sigma, H)$ is the direct sum of $t_{s}$ isomorphic principal indecomposable modules.

\section{BIBLIOGRA PHY}

1. B. G. Basmaji, Monomial representaions and metabelian groups, Nagoya Math. J. 35 (1969), 99-107. MR 39 \#5709.

2. R. Brauer, Zur Darstellungstheorie der Gruppen endlicher Ordnung. II, Math. Z. $72(1959 / 60), 25-46$. MR $21 \# 7258$.

3. - On blocks and sections in finite groups. I, Amer. J. Math. 89 (1967), 1115-1136. MR 36 \#2716.

4. - Some applications of the theory of blocks of characters of finite groups. IV, J. Algebra 17 (1971), 489-521.

5. A. H. Clifford, Representation induced in an invariant subgroup, Ann. of Math. (2) 38 (1937), 533-550.

6. C.W. Curtis and I. Reiner, Representation theory of finite groups and associative algebras, Pure and Appl. Math., vol. XI, Interscience, New York, 1962. MR 26 \#2519.

DEPARTMENT OF MATHEMATICS, CALIFORNIA STATE COLLEGE, LOS ANGELES, CALIFORNIA 90032 